\title{
Post-infarction left ventricular free wall rupture: 12-years experience from the Cardiac Centre of the Institute of Clinical and Experimental Medicine in Prague, Czech Republic
}

\author{
Petr Kacer ${ }^{a, b}$, Vera Adamkova ${ }^{b}$, Jaroslav Alois Hubacek ${ }^{b}$, Tereza Cervinkova ${ }^{b}$, Vaclav Adamek, Ivana Kralova Lesna ${ }^{b}$, \\ Vera Lanskab ${ }^{b}$ Jan Pirk ${ }^{b}$
}

Background. Post-infarction left ventricular free wall rupture (LVFWR) is a feared and catastrophic complication of myocardial infarction that carries a high surgical and hospital mortality. Due to the rarity of this complication, little information exists on surgical treatment and outcomes.

Goal and Methods. The goal of this study was to present our experience with LVFWR. We present a retrospective cohort of 19 consecutive patients who were surgically treated in the Cardiac Centre of the Institute of Clinical and Experimental Medicine in Prague between January 2006 and December 2017.

Results. Thirty-day mortality was $26 \%$. Five patients died. Four patients died in the operating theatre and one patient on the ninth postoperative day following re-rupture. Seventy-four percent of the patient cohort survived and were discharged from hospital. The median length of follow-up was 45 months (range 0.75-150). No patient died during follow-up. Median postoperative ejection fraction was $45 \%$ (range 25-65\%). Angina pectoris and dyspnea were investigated during follow-up and graded according to the Canadian cardiology society (CCS) and the New York Heart Association (NYHA) classifications. Fourteen patients had CCS class I, eight patients had NYHA class I dyspnea and six patients had NYHA class II. Re-rupture occurred after hospital discharge in one patient one month after the original surgery. The patient was treated successfully by urgent surgical intervention.

Conclusion. LVFWR is a catastrophic and challenging complication of myocardial infarction. Good outcomes can be achieved by rapid diagnosis and urgent surgical intervention as shown by our results.

Key words: acute myocardial infarction, myocardial rupture, ventricular wall

Received: March 12, 2020; Revised: April 12, 2020; Accepted: May 6, 2020; Available online: August 18, 2020

https://doi.org/10.5507/bp.2020.022

(c) 2021 The Authors; https://creativecommons.org/licenses/by/4.0/

${ }^{a}$ Department of Cardiac Surgery, University Hospital Kralovske Vinohrady, Third Faculty of Medicine, Charles University, Prague, Czech Republic

${ }^{b}$ Cardiac Centre, Institute of Clinical and Experimental Medicine, Prague, Czech Republic

Address for correspondence: Milos Taborsky: e-mail: milos.taborsky@fnol.cz

\section{INTRODUCTION}

Mechanical complications of myocardial infarction include rupture of the papillary muscles, the interventricular septum and the left ventricular free wall. Postinfarction left ventricular free wall rupture (LVFWR) is a rare cardiosurgical diagnosis that carries a high mortality, reported to range between 72 and $94 \%$ (ref. ${ }^{1}$ ). Cardiac rupture has been reported to complicate $6.4 \%$ (ref. ${ }^{1}$ ) of ST-elevation myocardial infarctions and has been shown to be declining in incidence, presumably due to the increase in the use of reperfusion therapy ${ }^{1}$. Post-infarction left ventricular free wall rupture was successfully treated surgically for the first time by FitzGibbon in 1971 (ref. $\left.{ }^{2}\right)$. In the literature there is little information on the surgical and long-term outcomes of PI-LVFWR. Due to its rarity, evidence-based medicine guidelines for LVFRW management do not yet exist. The goal of our study was to present our experience with LVFWR from a single institution over a 12-year period with a focus on surgical interventions and long-term follow-up.

\section{METHODS}

This was a retrospective study reporting all cases of post-infarction left ventricular free wall rupture treated surgically in the Cardiac Centre of the Institute of Clinical and Experimental Medicine in Prague, Czech Republic, during a 12 year period from 2006 to 2017. Patients were divided into two groups on the basis of in-hospital mortality: survivors $(n=14)$ and non-survivors $(n=5)$. Diagnosis of LVFWR was made by clinical examination, the findings of pericardial tamponade or effusions on echocardiography and electrocardiographic findings.

\section{Surgical procedures}

Several surgical procedures were used for the treatment of LVFWR. These techniques can be divided into two groups: procedures with ventriculotomy (Fig. 1) and procedures without ventriculotomy (Fig. 2). Procedures that involve ventriculotomy range from simply suturing the defect to complicated reconstructions using patches (Fig. 3) made from vascular prostheses or bovine pericardium. 


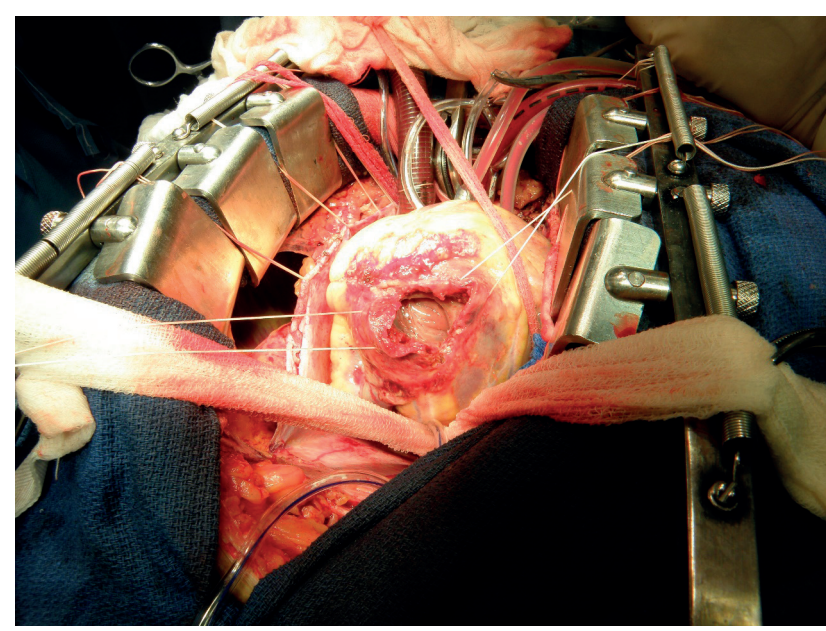

Fig. 1. Ventriculotomy in the middle of LVFWR.

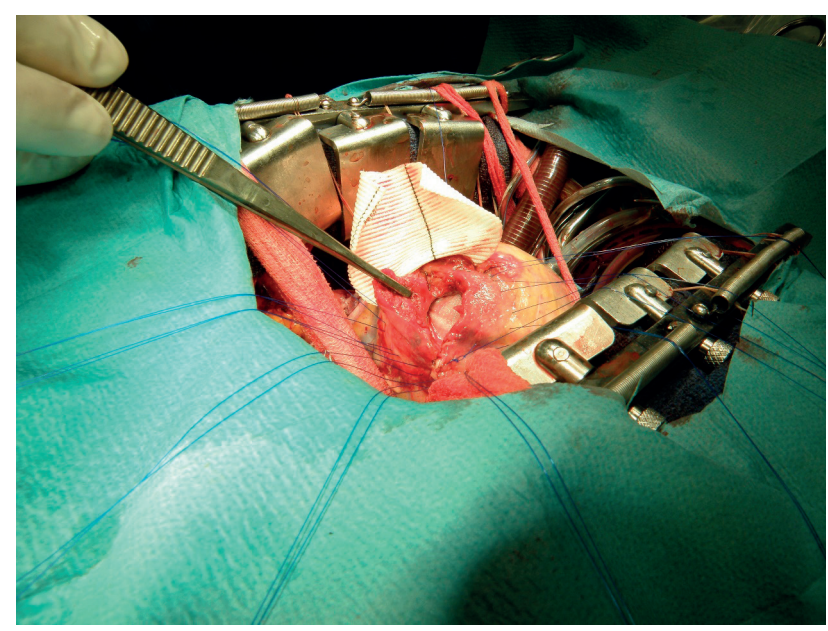

Fig. 3. Double-patch repair technique of LVFWR.

Surgical techniques that do not require ventriculotomy, known also as sutureless techniques (Fig. 4), use patches, such as TachoSil (Ethicon, USA) or bovine pericardium, to cover the ruptured area, and biological glues, such as Bioglue (Cryolife, USA) to fix the patches in place. It is necessary to assess the perioperative findings to choose the most suitable surgical technique. The choice of surgical technique depends on the type, size, and location of the rupture, the preference and experience of the surgeon and the hemodynamic state of the patient.

Techniques requiring ventriculotomy were usually used in patients with blow-out ruptures. These techniques are less sparing to the myocardium than sutureless techniques, as the sutures put tension on the myocardium surrounding the rupture. In contrast to techniques using ventriculotomy, cardiopulmonary bypass and cardioplegia are not needed for sutureless techqniues. Due to lack of data standardised protocols do not exist for selecting the surgical treatment of LVFWR. The current trend is to prefer simpler and more sparing techniques and as such sutureless techniques are gaining popularity.

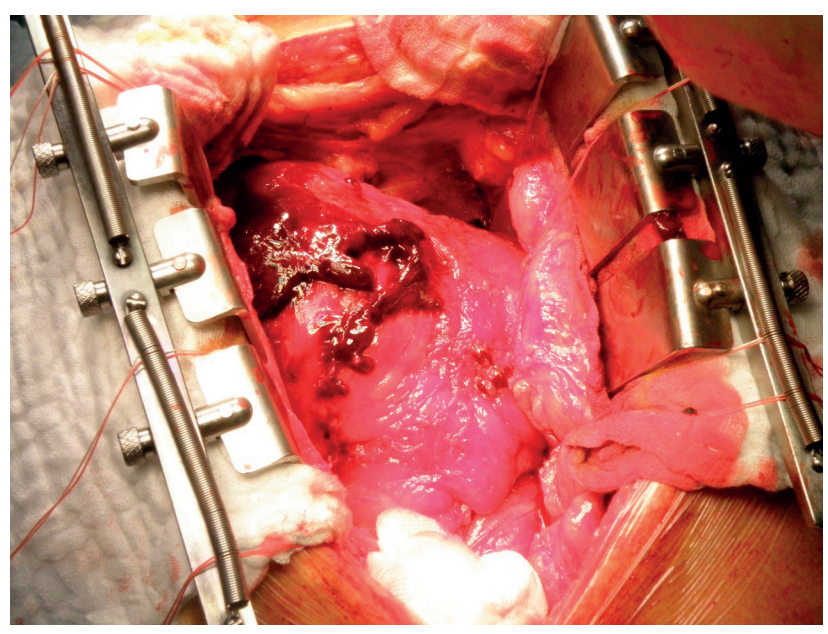

Fig. 2. LVFWR of anterior wall, will be repaired without ventriculotomy.

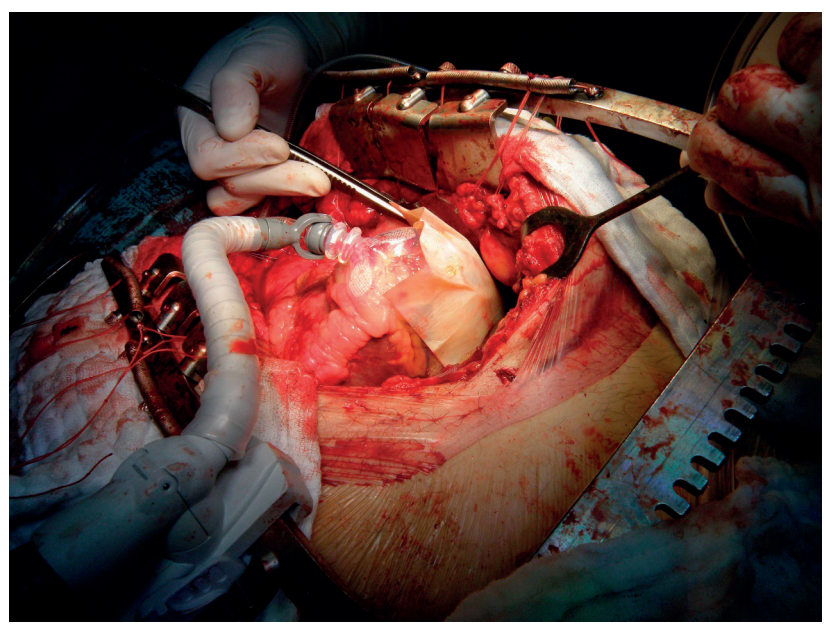

Fig. 4. Sutureless technique of repair of LVFWR.

\section{Statistical analysis}

Statistical analysis was performed with $\mathrm{JMP}^{\circledR}$ (2013 SAS Inc.). All continuous values were expressed as median with minimum and maximum and were compared with nonparametric Mann-Whitney tests. Categorical values were expressed as percentage and were compared with Fisher's exact tests. Kaplan-Meier analysis was used to analyse survival. All used tests were two-tailed.

\section{RESULTS}

\section{Clinical characteristics}

This cohort consists of 19 patients (12 males and 7 females) with a median age of 64 (range 48-79 years), who were operated between January 2006 and December 2017 for LVFWR. Twelve patients (63\%) had hypertension, nine patients $(47 \%)$ had hypercholesterolemia and two patients (10\%) had diabetes mellitus. Eight patients (42\%) were active smokers. Hypertension and hypercholesterolemia were more frequent in the non-survivors ( $86 \%$ vs. $48 \%$ and $64 \%$ vs. $36 \%$, respectively). This finding was 
Table. 1. Clinical characteristics.

\begin{tabular}{|c|c|c|c|c|}
\hline Clinical characteristics & Patients $n=19$ & $\begin{array}{c}\text { Survivors } \mathrm{n}=14 \\
(73.7 \%)\end{array}$ & $\begin{array}{l}\text { Non-survivors } \\
\mathrm{n}=5(26.3 \%)\end{array}$ & $P$ \\
\hline Number of males & $12(63.1 \%)$ & $9(64.3 \%)$ & $3(60.0 \%)$ & 1 \\
\hline Age (years) & $64(48-79)$ & $63(48-78)$ & $68(52-79)$ & 0.26 \\
\hline Body mass index & $27(20-34)$ & $26(20-34)$ & $30(27-33)$ & 0.089 \\
\hline Height & $172(148-186)$ & $171(148-186)$ & $172(168-174)$ & 0.91 \\
\hline Left ventricular ejection fraction & $40(20-64)$ & $40(20-64)$ & $40(20-45)$ & 0.7 \\
\hline Diabetes mellitus type 2 & $2(10.5 \%)$ & $1(20 \%)$ & $1(7.14 \%)$ & 0.47 \\
\hline Hypertension & $12(63 \%)$ & $8(57.1 \%)$ & $4(80 \%)$ & 0.6 \\
\hline Hypercholesterolemia & $9(47.3 \%)$ & $6(42.8 \%)$ & $3(60 \%)$ & 0.6 \\
\hline Significant stenosis of the arteries supplying the brain & $1(5.3 \%)$ & $1(7.14 \%)$ & $0(0 \%)$ & 1 \\
\hline Hemodynamically unstable & $10(52.6 \%)$ & $6(42.8 \%)$ & $4(80 \%)$ & 0.3 \\
\hline Cardiac tamponade & $9(47.3 \%)$ & $7(50 \%)$ & $2(40 \%)$ & 1 \\
\hline Cardiopulmonary resuscitation preoperatively & $7(38.8 \%)$ & $5(38.5 \%)$ & $2(40 \%)$ & 1 \\
\hline $\begin{array}{l}\text { Time interval between myocardial infarction and cardiac } \\
\text { rupture (hours) }\end{array}$ & $168(6.5-744)$ & $168(6.5-744)$ & $158(48-504)$ & 0.82 \\
\hline
\end{tabular}

Table 2. Perioperative characteristics.

\begin{tabular}{|c|c|c|c|c|}
\hline Perioperative characteristics & All patients $n=19$ & Survivors $n=14$ & Non-survivors $n=5$ & $P$ \\
\hline Surgical procedure & & & & 0.26 \\
\hline sutureless technique & $5(26.1 \%)$ & $5(35.7 \%)$ & $0(0 \%)$ & \\
\hline with ventriculotomy (suture, patch) & $14(73.6 \%)$ & $9(64.3 \%)$ & $5(100 \%)$ & \\
\hline Cardioplulmonary bypass & $17(89.4 \%)$ & $12(85.7 \%)$ & $5(100 \%)$ & 1 \\
\hline Length of cardiopulmonary bypass (minutes) & $125(65-315)$ & $113(65-225)$ & $170(95-315)$ & 0.14 \\
\hline Cross-clamp time ( $\mathrm{min})$ & $65(0-245)$ & $65(0-155)$ & $110(10-245)$ & 0.457 \\
\hline Concomitant cardiosurgical procedure & $11(57.9 \%)$ & $9(64.3 \%)$ & $2(40 \%)$ & 0.6 \\
\hline Intra-aortic balloon counterpulsion & $4(21 \%)$ & $2(14.3 \%)$ & $2(40)$ & 0.27 \\
\hline Extracorporeal membrane oxygenation & $1(5.3 \%)$ & $0(0 \%)$ & $1(20 \%)$ & 0.26 \\
\hline
\end{tabular}

not statistically significant $(P$ values of 0.6 and 0.6 , respectively). The median preoperative ejection fraction of the left ventricular was $40 \%$ (median $20-64 \%$ ), and there was not a significant difference between ejection fractions between the two groups $(P=0.7)$. Ten patients ( $53 \%)$ were hemodynamically unstable before the surgery. Cardiac tamponade occured in 9 patients (47\%), of whom two ( $40 \%)$ were in the group of non-survivors.

Cardiopulmonary resuscitation was performed in 7 patients (39\%), two of these patients were non-survivors. Three patients (16\%) had cardiogenic shock, two of which died after the surgery. Intra-aortic balloon counterpulsion was performed in one patient $(5 \%)$ peroperatively, who was in the survivors group. Extracorporeal membrane oxygenation was not used preoperatively in any patients. The median time between onset of the myocardial infarction and diagnosis of LVFWR was 168 hours (range 6.5744). There were no significant differences in preoperative characteristics between the two groups of patients (see Table 1).

Seventeen patients (89\%) underwent surgery with and two patients (11\%) without cardiopulmonary bypass. Fourteen patients (74\%) underwent procedures using ventriculotomy and all had cardiopulmonary bypass. Techniques using ventriculotomy included endoventricular plasty ( 7 patients, $42 \%$ ), direct suture (6 patients,
$32 \%$ ) and closure with a patch (1 patient, $5 \%$ ). Sutureless techniques (without ventriculotomy) were used in five patients $(26 \%)$, of whom 2 were operated on without cardiopulmonary bypass. All five patients who underwent sutureless procedures survived. There was no significant difference in the number of patients who underwent ventriculotomy procedures and sutureless procedures (14 vs. $5, P=0.26$ ). For a summary of the perioperative characteristic see Table 2.

Concomitant procedures were performed in 11 patients $(58 \%)$. The most common concomitant procedure was coronary artery bypass grafting, which was performed in 5 patients $(26 \%)$. Two patients $(11 \%)$ underwent closure of a ventricular septal defect. Two patients (11\%) underwent mitral valve repair; one also underwent closure of a ventricular septal defect. One patient (5\%) underwent extirpation of a myxoma of the left atrium and closure of an isolated defect of the interventricular septum (see Table 3).

The location of the rupture was identified intraoperatively. Nine patients (47\%) had posterior wall ruptures, six patients (32\%) had lateral wall ruptures and four patients ( $21 \%)$ had anterior wall ruptures. In the non-survivors two patients $(40 \%)$ had posterior wall ruptures, two (40\%) had lateral wall ruptures and two (20\%) had anterior wall ruptures (see Fig. 5). There was no statistically significant 
difference in rupture location between survivors and nonsurvivors $(P=0.89)$.

\section{Outcomes during hospitalization}

Fourteen patients (74\%) survived and five patients (26\%) died. Of the patients that survived nine were male and five were female. Of the patients that died three were male and two were female. Four patients died in the operating theatre. The cause of death in all cases was uncontrollable bleeding, in one case combined with cardiac failure. In this patient extracorporeal membrane oxygenation was used in the operative theatre. In all other cases mechanical cardiac support was not needed. One patient died on the ninth postoperative day following rerupture, which caused massive cardiac tamponade. This patient was resuscitated and underwent urgent reoperation. The intraoperative finding was surgically untreatable. Median blood loss was $490 \mathrm{~mL}$ (range 220-5450 mL). Four patients $(21 \%)$ underwent revision for postoperative bleeding, three (21\%) of which were in the group of survivors. Three patients (16\%) had renal complications, one of whom was in the group of non-survivors. The renal complications were renal insufficiency not requiring dialysis. Three patients (16\%) had postoperative delirium. The median length of stay on the ICU was $50 \mathrm{~h}$ (range 22-412). The median length of hospitalization was 7 days (range 0-61). For a summary of the outcomes during hospitalization see Table 4.

\section{Long-term outcomes}

None of the patients were lost to follow-up (Follow-up $100 \%)$ and none of the patients who were discharged died

Table 3. Concomitant surgery.

\begin{tabular}{lccc}
$\begin{array}{l}\text { Concomitant } \\
\text { surgery }\end{array}$ & $\begin{array}{c}\text { All patients } \\
\mathrm{n}=19\end{array}$ & $\begin{array}{c}\text { Survivors } \\
\mathrm{n}=14\end{array}$ & $\begin{array}{c}\text { Non-survivors } \\
\mathrm{n}=5\end{array}$ \\
\hline CABG all & 7 & 7 & \\
CABG only & 5 & 5 & \\
CABG + VSD & 2 & 2 & 1 \\
MVP + VSD + & 1 & & \\
$\quad$ ECMO & & & 1 \\
VSD & 1 & & \\
MVP & 1 & 1 & \\
Myxoma & 1 & 1 & \\
\hline
\end{tabular}

in the follow-up period (see Fig. 6, Kaplan Meier survival curves). The median follow-up period was 45 months, range 0.75 - 150). The median postoperative left ventricular ejection fraction was $45 \%$ (range $25-65 \%$ ), (see Fig. 7). We investigated whether the patients had angina pectoris or dyspnea during the follow-up period. Angina pectoris was graded using the Canadian Cardiovascular Society (CCS) classification and dyspnea was graded using the New York Heart Association (NYHA) classification. Fourteen (100\%) patients had CCS class I (see Fig. 8). Eight (57\%) patients had NYHA class I and six patients (43\%) had NYHA class II (see Fig. 9).

Re-rupture occurred in one patient one month postoperatively. This patient originally underwent sutureless repair of a rupture of the posterior wall of the left ventricle. The re-rupture was treated by endoventricular and mitral valve plasty. Extracorporeal membrane oxygenation was used. Blood loss reached 15, $440 \mathrm{~mL}$. The patient survived. Postoperatively the patient had an ejection fraction of $30 \%$, NYHA grade II dyspnea and does not have angina pectoris. One patient had such a large myocardial infarction that immediately after the operation (sutureless technique for an anterior wall rupture) was put on the waiting list for a heart transplant. After three months the patient underwent a successful heart transplantation.

\section{DISCUSSION}

Left-ventricular free wall rupture is a feared complicated of myocardial infarction with a poor prognosis. Shamsad et al. ${ }^{3}$ report $13.3-25 \%$ of deaths resulting from myocardial infarction are caused by LVFWR. Figueras et al. ${ }^{1}$ reported that cardiac rupture occurred as a complication of ST-elevation myocardial infarction in $6.2 \%$ of patients between the years 1977 and 1982 and 3.2\% between 2001 and 2006. The decrease of incidence is thought to be due to the implementation of reperfusion therapy, particularly direct percutaneous coronary intervention.

The main limitation of research on LVFWR is that it is a rare complication and that it is not possible to perform randomised studies. The number of published case series on LVFWR is low and contains small numbers of patients over long time periods. For example Pretre at al. ${ }^{4}$ reports on 5 patients over a 8 year period, McMullen et al. ${ }^{5} 18$

Table 4. Early results.

\begin{tabular}{|c|c|c|c|c|}
\hline Early results & Patients $n=19$ & Survivors $n=14$ & Non-survivors $n=5$ & $P$ \\
\hline Blood loss (ml, median, range) & $490(220-5450)$ & $445(220-5450)$ & $740(740-740)$ & 0.8 \\
\hline Revision for bleeding & 4 & 3 & 1 & \\
\hline \multicolumn{5}{|l|}{ Cause of death } \\
\hline bleeding & & & $2(40 \%)$ & \\
\hline heart failure & & & $2(40 \%)$ & \\
\hline bleeding and heart failure & & & $1(20 \%)$ & \\
\hline ICU stay (hours, median, range) & $50(22-412)$ & $47(22-412)$ & $116(116-116)$ & 0.49 \\
\hline Hospital stay (days, median, range) & $7(0-61)$ & $10(6-61)$ & $0(0-9)$ & 0.009 \\
\hline Renal complications (no dialysis) & $3(16 \%)$ & $2(14 \%)$ & $1(20 \%)$ & \\
\hline Neurological complications & $3(16 \%)$ & $3(21 \%)$ & 0 & \\
\hline
\end{tabular}




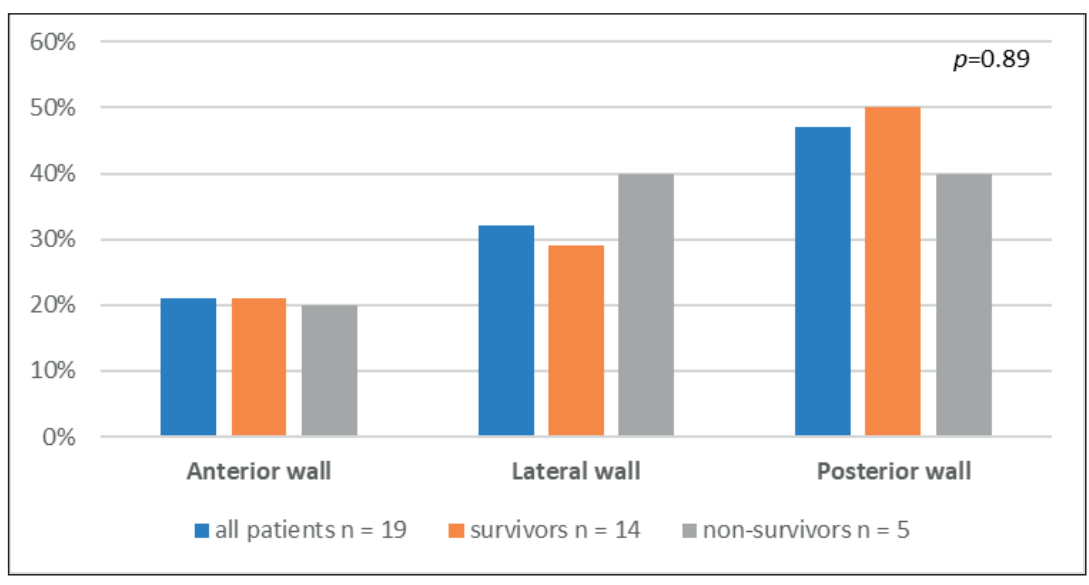

Fig. 5. Site of LV rupture.

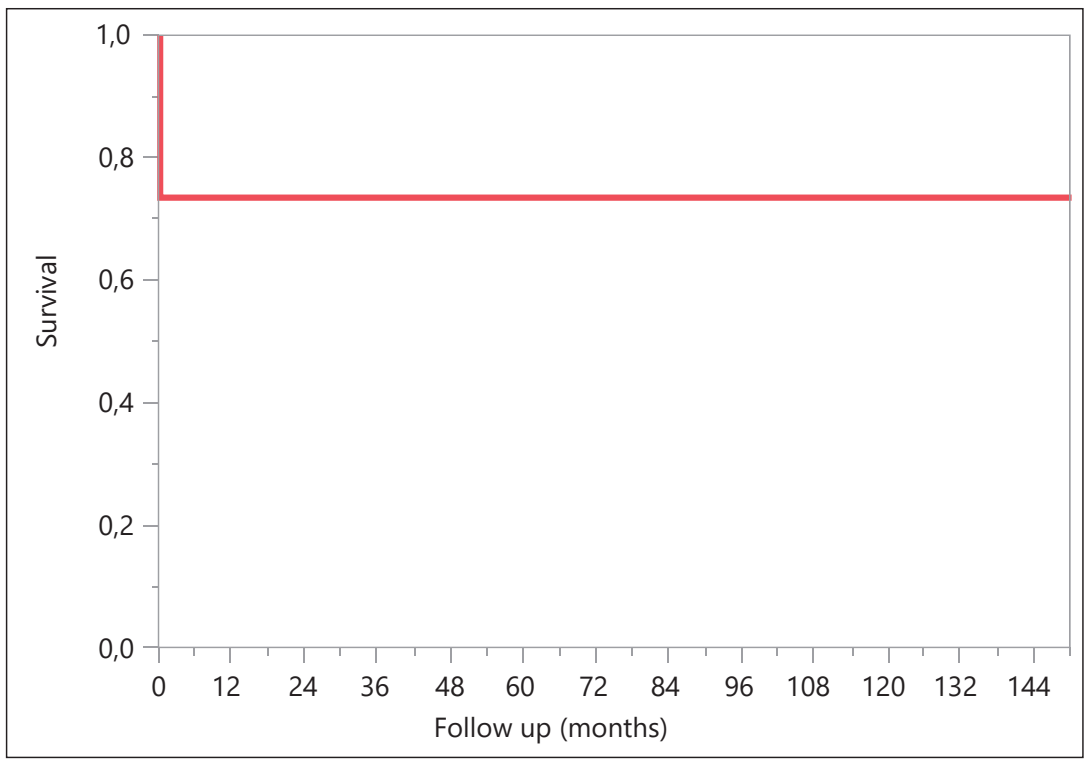

Fig. 6. Kaplan Meier survival curves.

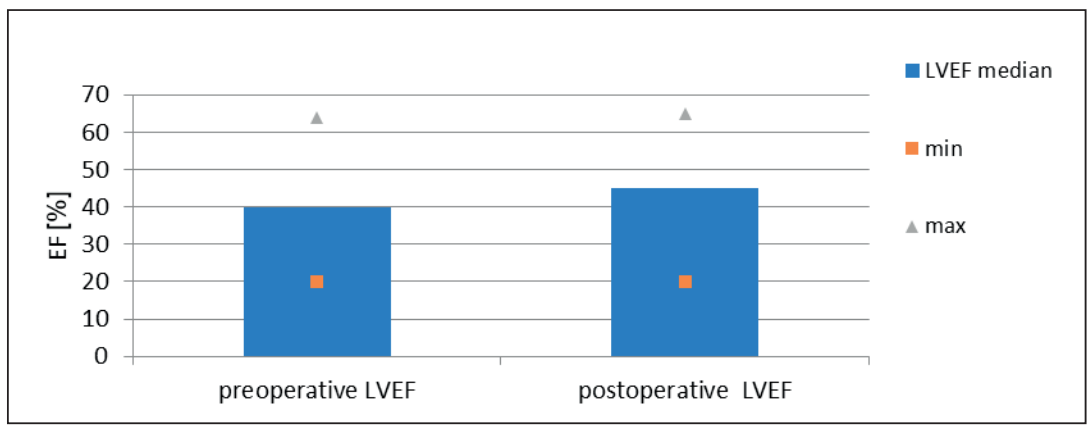

Fig. 7. Preoperative and postoperative left ventricular ejection fractions of survivors.

patients over 12 years, Lemura et al. ${ }^{6} 19$ patients over 12 years, Lachapell et al. 5 patients of 9 years. Sakaguchi et al. ${ }^{8} 32$ patients over 14 years and Formica et al. ${ }^{9} 35$ patients over 17 years. Our cohort of 19 patients ranks among one of the largest case series of patients from a single institution.

The in-hospital mortality of LVFWR over the last three decades has been very high, ranging between 72 and $94 \%$ (ref. $^{1}$ ). In our cohort $26 \%$ of the patients died during hospitalization. In total five patients died, four of which died in the operating theatre. A high mortality has been associated with LVFWR by other studies, McMullen et al..$^{5}$ report a mortality of $61 \%$ in a cohort of 18 patients, Sakaguchi et al. ${ }^{8}$ report a mortality of $15.6 \%$ in a cohort of 32 patients, all of whom underwent sutureless procedures, and Formica et al. ${ }^{9}$ report a mortality $34 \%$ in a cohort of 35 patients. 


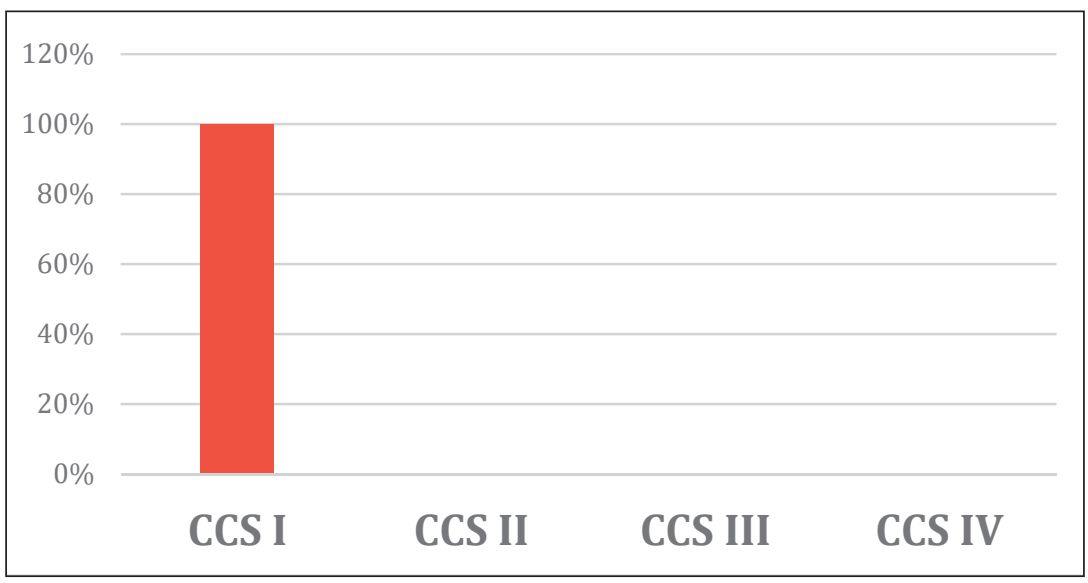

Fig. 8. Postoperative Angina Pectoris according CCS.

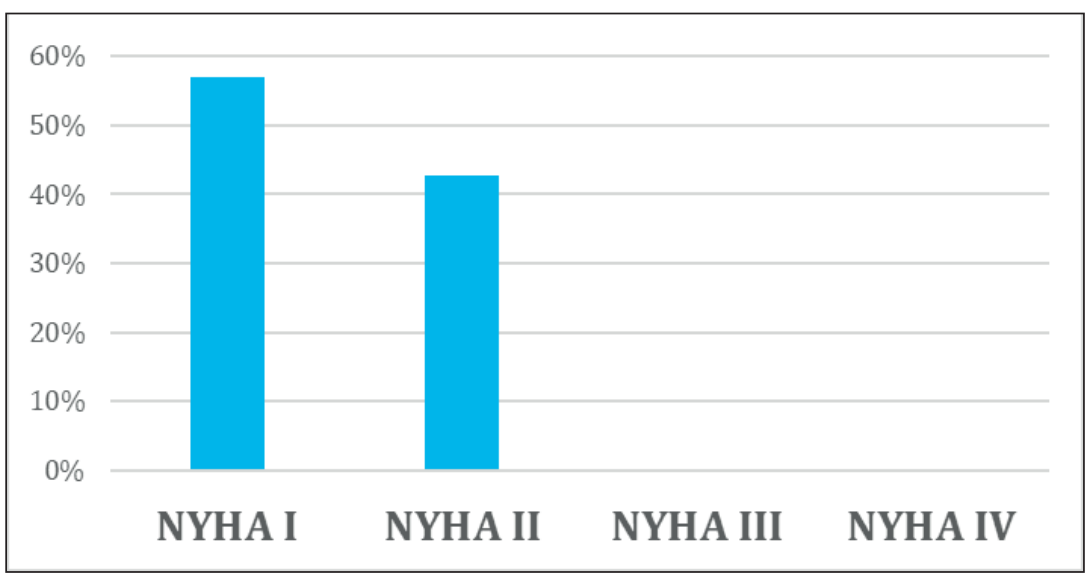

Fig. 9. Postoperative Dyspnea according NYHA.

In our cohort the majority of the patients were male $(63 \%)$, which contrasts to the traditional view that the LVFWR typically occurs in females, above 60 years of age, as a complication of their first myocardial infarction, in non-hypertrophied hearts. The majority of our patients had hypertension (63\%) and hypertension was more frequent in the group of non-survivors (80\%). This result was not statistically significant. Hypertension, when present at the time of the myocardial infarction is a risk for development of cardiac rupture. However, long-term hypertension is protective of cardiac rupture as it leads to myocardial hypertrophy ${ }^{10-13}$.

Preoperative hemodynamic state is a strong prognostic factor. In our cohort cardiopulmonary resuscitation was necessary in 7 patients (39\%) of whom two (40\%) were non-survivors. Three patients (16\%) had cardiogenic shock, two of which died after the procedure. In Formica et al.'s cohort preoperative heart failure was identified as an independent risk factor for hospital mortality and no evidence was given that perioperative extracorporeal membrane oxygenation influence survival ${ }^{9}$. In our cohort extracorporeal membrane oxygenation was not used preoperatively and the one patient it was used in intraoperatively died.

We used a wide spectrum of surgical techniques for reconstructing the left ventricle, which were arbitrarily divided into two groups: procedures with ventriculotomy and procedures without ventriculotomy. Procedures without ventriculotomy consisted of sutureless techniques, which can be used in cases of "oozing types" of rupture. There was no significant difference in the number of patients in each of the two groups of procedures $(P=0.26)$, presumably due to the overall small number of patients. All patients who underwent sutureless techniques survived. We presume this to be due to the fact that the patients requiring procedures with ventriculotomy have worse morphological findings at surgery, more frequently manifest with blow-out ruptures and usually are hemodynamically less stable.

One of the most serious postoperative complications of LVFWR surgery is re-rupture. Various incidences of this complication have been reported in the literature. In McMullen et al.'s report of 18 patients who underwent surgery for LVFWR eleven patients died, four as a result of re-rupture ${ }^{5}$. In Sakaguchi's cohort of 32 patients with LVFWR treated by sutureless techniques. They applied autologous pericardium fixed with biological glue to cover the defects. The in-hospital mortality in their cohort was $15.6 \%$. Re-rerupture occurred in forty percent of the patients who died. During the follow-up period after discharge no further cases of re-rupture occurred. Left ventricular Aneurysms formed in five patients, and 
a pseudoaneurysm formed in one patient ${ }^{8}$. In our cohort re-rupture occurred in two patients $(10.5 \%)$. In one patient the re-rerupture occurred on the ninth postoperative day and manifested as massive tamponade. The patient was resuscitated and underwent urgent surgical revision. The intraoperative finding was surgically untreatable and the patient died. The original rupture was located on the posterior wall of the left ventricle and was treated by the double-patch technique, in which two patches are used to close the defect, one on the internal and one external surface of the ventricle. This technique is one of the most complicated and extensive procedures for LVFWR. In the other patient in whom re-rupture occurred a sutureless procedure was originally performed for a posterior wall rupture. The re-rupture occurred one month later and the patient underwent an urgent endoventricular plasty of the left ventricle together with a mitral valve repair. Extracorporeal membrane oxygenation was temporarily used intraoperatively and the patient survived. It is not clear if some techniques are more frequently associated with re-rerupture than others. We postulate that sutureless techniques may be more frequently associated with re-rupture than ventriculotomy procedures. However, at the time of writing no strong evidence exists to back up this hypothesis. Zoffoli et al. ${ }^{14}$, however, present data in contrast to this. They published a cohort of 25 patients who underwent "patch a glue", i.e. a sutureless technique and no cases of re-rupture occurred in the follow-up period.

The median preoperative ejection fraction in our cohort of patients was $40 \%$ (range $20-64 \%$ ) and the difference in preoperative ejection fraction between the patients who died and the patients who survived was not statistically significant. It is important to state that all preoperative ejection fractions were measured after the onset of the myocardial infarction. Pre-infarction ejection fractions, which can be compared with preoperative postinfarction ejection fractions to predict the size of the infarction, were not available. From this we can deduce that the patients did not undergo any significant worsening of state of health before the infarctions that led to them to seek medical help.

In our cohort there was no statistically significant difference between the postoperative and preoperative ejection fractions $(P=0.55)$, the values were very similar (see Figure 7 ). The reason for this may be that the extensive reconstructive procedures do not necessarily lead to iatrogenic destruction of healthy myocardium. We presume that the surgical reconstruction of the left ventricle does not necessarily worsen the functionality of the ventricle and lead to a lower ejection fraction.

One-hundred-percent of the patients had CCS grade I angina pectoris and no patients had CCS class II, III or IV angina pectoris. This can be explained by the high incidence of ST-elevation infarctions in our cohort, which led to transmural necrosis of the myocardium supplied by the affected vessel. Angina pectoris can not occur in dead myocardium and the other vessels were either healthy or were revascularized during the operation.

\section{Study limitations}

The study is limited by its small size of only 19 patients and its retrospective design. Larger cohorts of patients are needed to answer several questions concerning LVFWR, such as what are the factors predict outcome and what is the relationship between the different surgical techniques and outcomes.

\section{CONCLUSION}

LVFWR is a catastrophic, devastating and difficult to predict complication of myocardial infarction. The only treatment is urgent surgery, without which its mortality is very high. Patients with LVFWR have a good chance to survive diagnosed early and treated by urgent surgery. Several different surgical techniques exist for the treatment of LVFWR, which differ in invasiveness and extent to which they harm the myocardium, which is already damaged by infarction and rupture. Our patients who were successfully treated did not have significantly impaired postoperative ejection fractures and had good clinical outcomes. Furthermore, none of the patients who survived the immediate postoperative period and were discharged from hospital died during the follow-up period.

Acknowledgement: The authors would like to thank dr. Adam Whitley for translation of the manuscript. The work was supported by the grant AZV MZ ČR No 16-28352A Author contribution: Authors contributed equally to this manuscript.

\section{REFERENCES}

1. Figueras J, Alcalde O, Barrabes A, Serra V, Alguersuari J, Cortadellas J, Lidón RM. Changes in hospital mortality rates in 425 patients with acute ST- elevation myocardial infarction and cardiac rupture over a 30-year period. Circulation 2008;118:2783-9.

2. Fitzgibbon G M, Hooper Gd Ha. Heggtveit Successful surgical treatment of post cardiac external cardiac rupture. J Thorac Cardiovasc Surg 1972;63:622-30.

3. Shamshad F, Kenchaiah S, Finn PV, Soler-Soler J, Mcmurray Jj, Velasquez Ej, Maggioni Ap, Califf Rm, Swedberg K, Kober L, Belenkov Y, Varshavsky S, Pfeffer Ma, Solomon Sd. Fatal myocardial rupture after acute myocardial infarction complicated by heart failure, left ventricular dysfunction, or both: the VALsartan In Acute myocardial iNfarcTion Trial (VALIANT). Am Heart J 2010;160:145-51.

4. Pretre R, Line A, Jenni R, Turina M. Surgical treatment of acquired left ventricular pseudoaneurysms. Ann Thorac Surg 2000;70:553-7.

5. McMullan MH, Maples Md, Kilgore TI Jr, Hindman Sh. Surgical experience with left ventricular free wall rupture. Ann Thorac Surg 2001;71:1894-9.

6. lemura J, Oku H, Otaki M, Kitayama H, Inoue T, Kaneda T. Surgical strategy for left ventricular free wall rupture after acute myocardial infarction. Ann Thorac Surg 2001;71:201-4.

7. Lechapelle K, De Varennes B, Ergina PI, Cecedo R. Sutureless patch technique for left ventricular rupture postinfarction. Ann Thorac Surg 2002;74:96-101.

8. Sakaguchi G, Komiya T, Tamura N, Kobayashi T. Surgical Treatment for Postinfarction Left Ventricular Free Wall Rupture. Ann Thor Surg 2008;85:1344-6.

9. Formica F, Mariani, Singh G, D’Alessandro S, Messina La, Oluwaseun N, Bamodu A, Sangalli Fabio, Paolini G. Postinfarction left ventricular free wall rupture: a 17-year single-center experience. Eur J Cardiothorac Surg 2018;53:150-6. doi:10.1093/ejcts/ezx271 
10. Lopez-Sendon J, Gurfinkel Ep, Lopez De Sa E, Agnelli G, Gore Jm, Steg Pg, Eagle Ka, Cantador Jr, Fitzgerald G, Granger Cb. Factors related to heart rupture in acute coronary syndromes in the Global Registry of Acute Coronary Events. Eur Heart J 2010;31:1449-56.

11. Mg Abrignani, Dominguez Lj, Biondo G, Di Girolamo A, Novo G, Barbagallo M, Braschi A, Braschi G, Novo S. In-hospital complications of acute myocardial infarction in hypertensive subjects. Am J Hypertens 2005;18:165-70.

12. Widmer A, Line A Z, Attenhofer Jost Ch, Buergi B, Brunner - La Rocca Hp, Salomon F, Seifert B, Jenni R. Mechanical complications after myocardial infarction reliably predicted using C- reactive protein levels and lymphocytopenia. Cardiology 2003;99:25-31.

13. Becker Rc, Charlesworth A, Wilcox Rg, Hampton J, Skene A, Gore Jm, Topol Ej. Cardiac rupture associated with thrombolytic therapy: the impact of time to treatment in the Late Assessment of Thrombolytic Efficacy (LATE) study. J Am Coll Cardiol 1995;25:1063-8.

14. Zoffoli G, Battaglia F, Venturini A, Asta A, Terrine A, Zanchettin Ch, Mangin D. A Novel Approach it Ventricular Rupture: Clinical Needs and Surgical Technique. Ann Thorac Surg 2012;93:1002-3. 